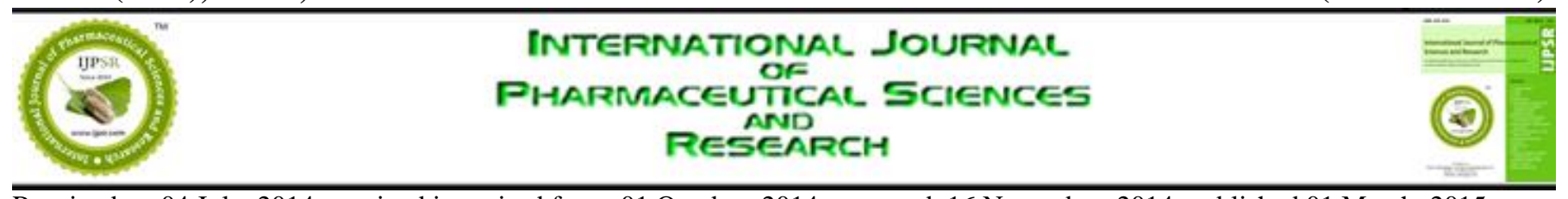

Received on 04 July, 2014; received in revised form, 01 October, 2014; accepted, 16 November, 2014; published 01 March, 2015

\title{
APOPTOSIS: BASIC CONCEPTS, MECHANISMS AND CLINICAL IMPLICATIONS
}

\author{
Bibhas Kar and S. Sivamani
}

Centre for Genetic Studies \& Research, The Madras Medical Mission, Chennai-600037,TN, India.

Keywords:

Apoptosis,

Intrinsic/Extrinsic Pathway, Anti

Apoptotic Genes, Granzyme/Perforin

\section{Correspondence to Author:}

Dr. Bibhas Kar

Consultant \& Head

Centre for Genetic Studies \&

Research, The Madras Medical

Mission Chennai - 600037, TN,

India.

E-mail: drbibhas_kar@yahoo.co.in

ABSTRACT: Apoptosis, a well choreographed gene-directed cellular destruction, plays an important role in a variety of biological events, including morphogenesis, homeostatic maintenance of various tissues and removal of harmful cells while dysregulated apoptosis has been implicated in a variety of disease such as cancer, systemic and organ specific autoimmune disease and neurodegenerative disorders. Apoptosis is initiated by extracellular or intracellular signals in which complex machinery is activated to start a cascade of events ultimately leading to the degradation of nuclear DNA and dismantling of the cell. Cellular disruption results from the activation of a family of cysteine proteases known as caspases. Caspases are synthesized as inactive proenzymes, which upon activation cleave various substrates in the cytoplasm or nucleus leading to changes in morphological and cell death. The goal of this review is to provide a general overview of basic knowledge on the process of apoptosis including morphology, characterization, assays, anti apoptotic genes, mechanisms and relevance to diseases.

INTRODUCTION: Apoptosis or programmed cell death plays a crucial role in many biologic processes and is involved in various diseases. The term is derived from the Greek word, meaning a plant shedding leaves 1,2 . Apoptosis, a well choreographed gene-directed cellular destruction, plays an important role in a variety of biological events, including morphogenesis, homeostatic maintenance of various tissues and removal of harmful cells while dysregulated apoptosis has been implicated in a variety of disease such as cancer, systemic and organ specific autoimmune disease and neurodegenerative disorders ${ }^{3,4}$.

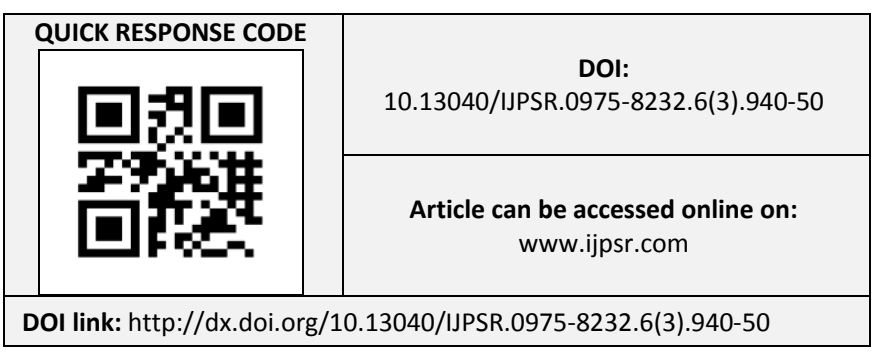

Apoptosis occurs: (1) during development and aging (2) removal of excessive of potentially dangerous cells (3) tumor cells (4) virus infected cell. Apoptosis also occurs as a defense mechanism such as in immune reactions or when cells are damaged by disease or noxious agents ${ }^{5}$. In this paper we review outline mechanisms of apoptosis and clinical implication.

\section{Apoptotic Process:}

Apoptotic process consists of three steps. In the first step, intracellular and extracellular signals activate specific pathways that will determine whether a cell should live or die. The tumour suppressor gene p53 is involved in a checkpoint that decides whether a cell progresses in the cell cycle or dies by apoptosis in response to deoxyribonucleic acid (DNA) damage. Interaction of Fas ligand or tumour necrosis factor (TNF) with their membrane receptor increases intracellular ceramide level. Growth factors or interactions with 
extracellular matrix increase intracellular calcium and activate the protein kinase $\mathrm{C}$ (PKC). The second step involves two checkpoints that could regulate the final cell death pathway. One checkpoint is controlled by proteins from the Bcl-2 family. Bcl-2 expression can be increased by interaction with viral proteins or mimicked by LMP-1 from Epstein-Barr virus. The other checkpoint could be determined by the cysteine proteases related to interleukin-1 $\beta$ (IL- $1 \beta$ ) converting enzyme (ICE) that is inhibited by the CrmA protein from cowpox virus. The cell death pathway then reaches a point of no return, and apoptosis is associated with internucleosomal DNA fragmentation, proteolysis of several nuclear and cytosolic proteins, and characteristic morphological changes ${ }^{6}$ (Fig 1).

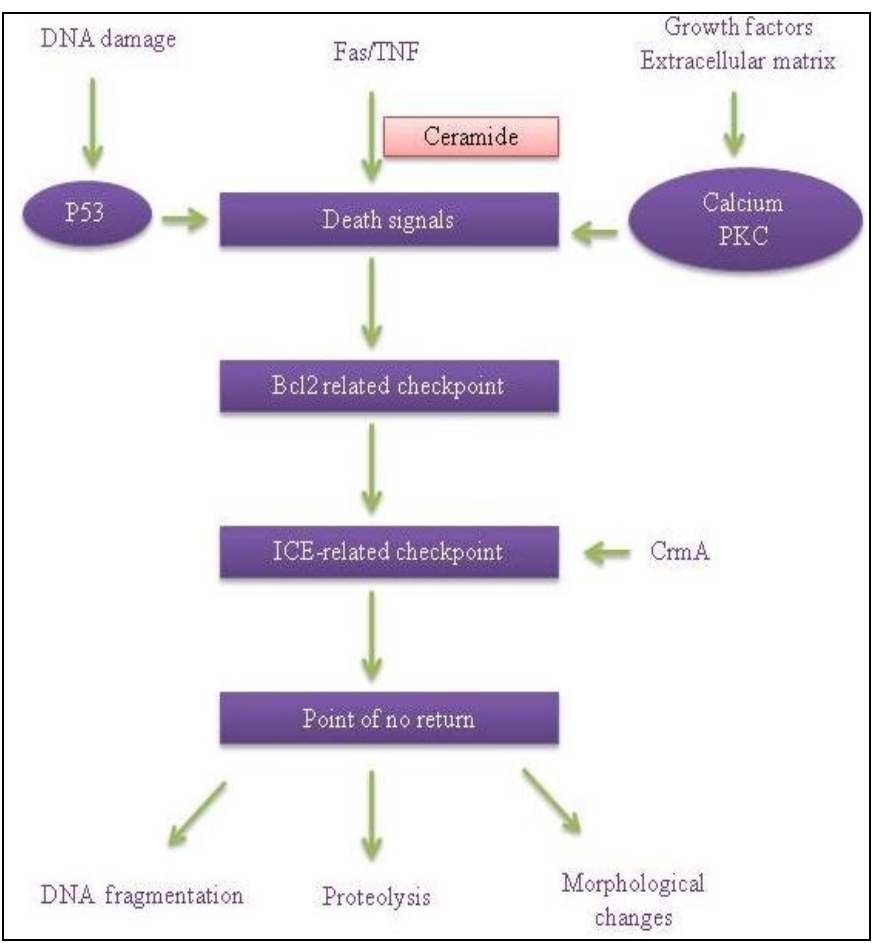

FIGURE 1: HYPOTHETICAL MODEL OF CELL DEATH REGULATION IN APOPTOSIS.

\section{Morphology of apoptosis:}

Light and electron microscopy have identified the various morphological changes that occur during apoptosis ${ }^{7}$. During the early process of apoptosis, cell shrinkage and pyknosis are visible by light microscopy ${ }^{8}$. At cell shrinkage, the cells are smaller in size, the cytoplasm is dense and the organelles are more tightly packed. Pyknosis is the result of chromatin condensation and this is the most characteristic feature of apoptosis.
Histological examination of apoptosis with hematoxylin and eosin stain, indicate which involves in single cells or small clusters of cells. The apoptotic cell appears as a round or oval mass with dark eosinophilic cytoplasm and dense purple nuclear chromatin fragments. Electron microscopy can better define the sub-cellular changes. Extensive plasma membrane blebbing occurs followed by karyorrhexis and separation of cell fragments into apoptotic bodies during a process called "budding". Apoptotic bodies consist of cytoplasm with tightly packed organelles with or without a nuclear fragment.

The organelle integrity is still maintained and all of this is enclosed within an intact plasma membrane. These bodies are subsequently phagocytosed by macrophages, parenchymal cells, or neoplastic cells and degraded within phagolysosomes. Macrophages that engulf and digest apoptotic cells are called "tingible body (bits of nuclear debris from the apoptotic cells) macrophages" and are frequently found within the reactive germinal centers of lymphoid follicles or occasionally within the thymic cortex.

\section{Characterization of apoptosis:}

Apoptosis is characterized by specific phenomenology; First, Condensation of cell nucleus and degradation of nuclear DNA via endonuclear cleavage of chromosome DNA, which breaks first into large $(50 \sim 300 \mathrm{~kb})$ and then into very small fragments ${ }^{9}$. Second, In the case of apoptosis, cell death is suicidal because cells execute an internal program of their death including activation of endogenous proteases, protein degrading enzymes ${ }^{10}$.

\section{Mechanisms of apoptosis:}

There are two distinct pathways that can initiate the caspase cascade, resulting in apoptosis: the intrinsic (or mitochondrial) pathway and the extrinsic (or death receptor) pathway ${ }^{11,12}$. There is an additional pathway that involves T-cell mediated cytotoxicity and perforin granzyme dependent killing of the cell. The perforin/granzyme pathway can induce apoptosis via either granzyme B or granzyme A. The extrinsic, intrinsic, and granzyme B pathways converge on the same terminal, or execution pathway. This pathway is initiated by the cleavage 
of caspase-3 and results in DNA fragmentation, degradation of cytoskeletal and nuclear proteins, cross-linking of proteins, formation of apoptotic bodies, expression of ligands for phagocytic cell receptors and finally uptake by phagocytic cells. The granzyme a pathway activates a parallel, caspase-independent cell death pathway via single stranded DNA damage ${ }^{13}$.

\section{Extrinsic Pathway:}

The extrinsic signalling pathways that initiate apoptosis involve transmembrane receptormediated interactions. This pathway is activated by death receptor (including Fas, TNFR1, DR4 and DR5) stimulation after the binding of death ligands (FasL, TNFa, TRAIL, and TNFSF10) ${ }^{14}$. The death receptors are characterized by intracellular motifs capable of homotypic interaction, including the death domain (DD) and the death effector domain (DED). The binding of the ligand, for example FasL, to the death receptor reorganizes inactive Fas complexes and stimulates the recruitment of adaptor proteins such as the Fas-associated death domain (FADD).

The adaptor protein FADD contains two interaction motifs: the DD domain which interacts with the DD domain of Fas receptor, and the DED which facilitates the recruitment of procaspase-8 (or -10) and the formation of the death-inducing signalling complex (DISC). The DISC formation leads to autocatalytic activation of caspase- 8 which subsequently cleaves and activates downstream effector caspase-3 15, 16 . Once caspase- 8 is activated, the execution phase of apoptosis is triggered (Fig. 2).

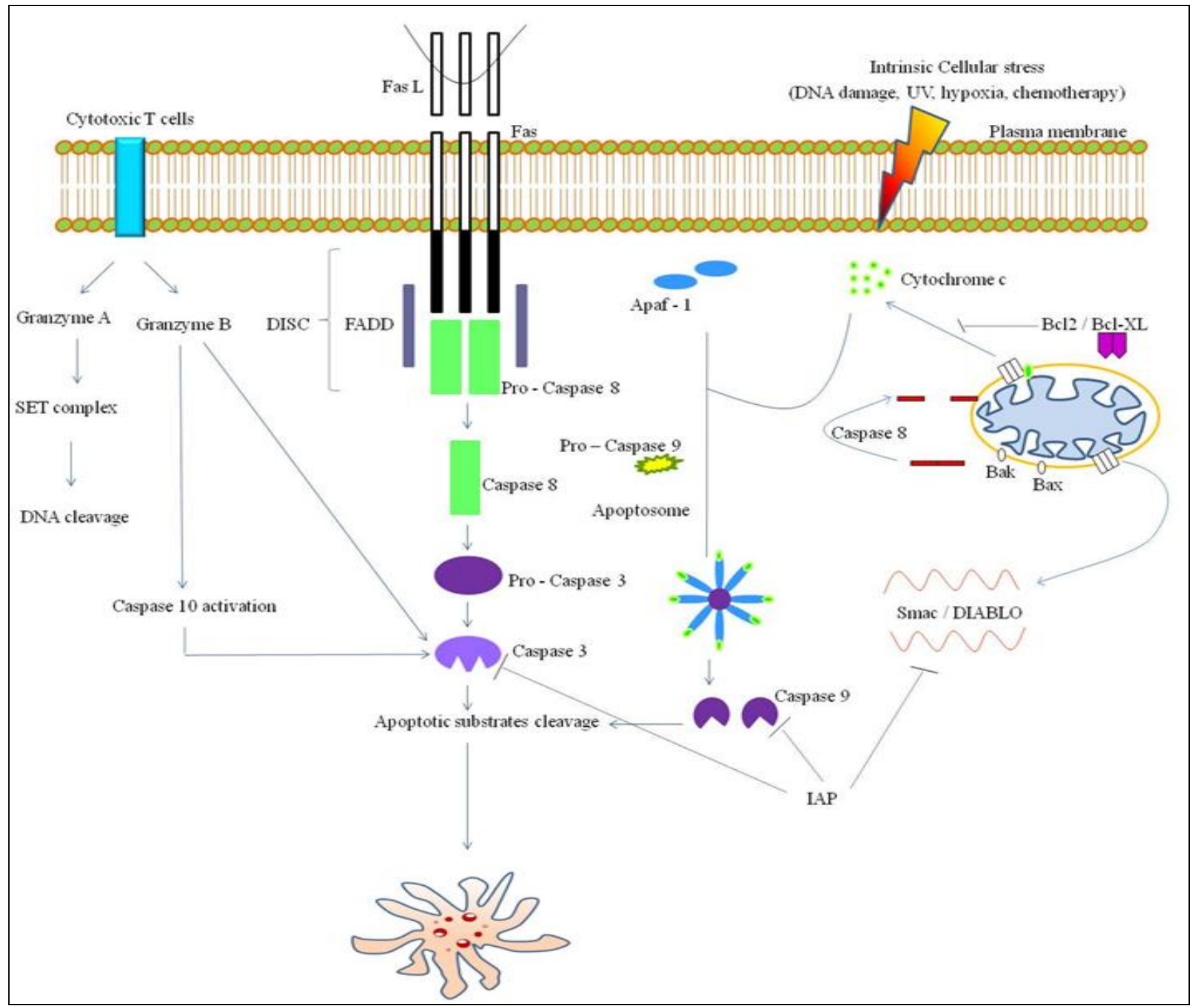

FIGURE 2: SCHEMATIC REPRESENTATION OF APOPTOTIC EVENTS. 


\section{Intrinsic Pathway:}

The intrinsic apoptotic pathway, which is mediated by the mitochondria, is activated by many extracellular and intracellular stimuli including loss of survival/trophic factors, toxins, radiations, hypoxia, oxidative stress, ischemia reperfusion and DNA damage ${ }^{17}$. The mitochondria outer membrane permeabilization (MOMP) is an important event in the apoptotic intrinsic pathway; the Bcl-2 family members have a major role in this permeabilization acting on the outer mitochondrial membrane. The death signals cause the proapoptotic Bcl-2 proteins to allow cytochrome c to leak out of the mitochondria.

The released cytochrome $\mathrm{c}$ and apoptotic proteaseactivating factor 1 (Apaf-1) bind to caspase 9, which then activates the caspase cascade, leading to cell death. Also, the induction of the pro-apoptotic BH3-only domain proteins [Bid, Bad, Noxa and p53-up-regulated modulator of apoptosis (PUMA)] can relay the signals to the mitochondria. Once induced, these proteins can facilitate the assembly of pro-apoptotic Bax and Bak into the pores in the outer mitochondrial membrane ${ }^{18}$. The process involves changes in mitochondrial permeability and release of various factors involved in apoptosis, including cytochrome $\mathrm{c}$ and apoptosis-inducing factor. The MOMP causes Cyt c release into the cytoplasm, where, in the presence of dATP, it induces the formation of the Apaf1-containing macromolecular complex known as apoptosome. This complex binds and promotes the proteolytic activation of procaspase-9. The mature caspase- 9 remains bound to the apoptosome where it recruits and activates the executioner caspase-3 and/or $-7^{19}$.

In addition to Cyt $\mathrm{c}$ release, the induction of MOMP leads to the cytosolic release of other essential pro-apoptotic molecules such as Smac/Diablo and Omi/ AtrA2, which can enhance apoptotic execution by inactivating the inhibitors of apoptotic proteins (IAPs). Under unstressful conditions, in fact, the caspases are inhibited by the IAPs; of these XIAP (X-linked inhibitor of apoptosis) protein is thought to be the most efficient: it binds to active forms of caspase-3, -7 and -9 and directly inhibits their proteolytic activity ${ }^{20,21}$. Intrinsic and extrinsic (or death receptor) have been well described that involve a number of proteins (Table 1 and $\mathbf{2}$ ).

\section{TABLE 1: PROTEINS INVOLVED IN APOPTOSIS INTRINSIC PATHWAY ${ }^{58,59}$}

\begin{tabular}{cll}
\hline \multicolumn{1}{c}{ Proteins } & Abbreviation & Alternate Nomenclature \\
\hline Apoptotic protease activating factor & Apaf-1 & APAF1 \\
Apoptosis inducing factor & AIF & Programmed cell death protein 8, mitochondrial \\
B-cell lymphoma protein 2 & Bcl-2 & Apoptosis regulator Bcl-2 \\
BCL2 like 1 & Bcl-x & BCL2 related protein \\
BCL2 related protein, long isoform & Bcl-XL & BCL2L protein, long form of Bcl-x \\
BCL2 related protein, short isoform & Bcl-XS & - \\
BCL2 like 2 protein & Bcl-w & Apoptosis regulator BclW \\
BCL2 associated athano gene & BAG & BAG family molecular chaperone regulator \\
BCL 2 associated X protein & BAX & Apoptosis regulator BAX \\
BCL2 antagonist killer 1 & BAK & BCL2L7, cell death inhibitor 1 \\
BCL2 antagonist of cell death & BAD & BCL2 binding protein, BCL2L8, BCL2 binding \\
& & component 6, BBC6, Bcl-XL/Bcl-2 associated \\
& BIM & death promoter \\
BCL2 interacting protein & BIK & NBK, BP4, BIP1, apoptosis inducing NBK \\
BCL2 interacting killer & Bcl-10 & mE10, CARMEN, CLAP, CIPER \\
B-cell lymphoma protein 10 & BID & p22 BID \\
BH3 interacting domain death agonist & Blk & B lymphoid tyrosine kinase, p55-BLK, \\
Bik-like killer protein & & MGC10442 \\
IAP & XIAP, API3, ILP, HILP, HIAP2, cIAP1, API1, \\
Inhibitor of Apoptosis Proteins & & MIHB, \\
& Nyc & c-myc, Myc proto-oncogene protein \\
Oncogene Myc & Caspase-9 & ICE-LAP6, Mch6, Apaf-3 \\
Second mitochondrial activator of caspases/direct & Smac/DIABLO & - \\
IAP binding protein with low PI & & \\
\hline
\end{tabular}


TABLE 2: PROTEINS INVOLVED IN APOPTOSIS EXTRINSIC PATHWAY ${ }^{58,59}$

\begin{tabular}{ccc}
\hline Proteins & Abbreviation & Alternate Nomenclature \\
\hline Cysteinyl aspartic acid protease 8 & Caspase-8 & FLICE, FADD-like Ice, MACH-1, MCH5 \\
Apo2 ligand & Apo2L & TRAIL/TNFSF10 \\
Apo3 ligand & Apo3L & TWEAK/TNFSF12/DR3LG \\
Death receptor 3 & DR3 & TNFRSF12, Apo3, WSL-1, TRAMP, LARD, DDR3 \\
Death receptor 4 & DR4 & TNFRSF10A, TRAILR1, APO2 \\
Death receptor 5 & DR5 & TNFRS10B, TRAIL-R2, TRICK2, KILLER, ZTNFR9 \\
Death effector domain & DED & Apoptosis antagonizing transcription factor, CHE1 \\
Fatty acid synthetase ligand & FasL & Fas ligand, TNFSF6, Apo1, apoptosis antigen ligand 1, \\
& & CD95L, CD178, APT1LG1 \\
Fatty acid synthetase receptor & FasR & Fas receptor, TNFRSF6, APT1, CD95 \\
Fas-associated death domain & FADD & MORT1 \\
Receptor-interacting protein & RIP & RIPK1 \\
Tumor necrosis factor alpha & TNF- $\alpha$ & TNF ligand, TNFA, cachectin \\
TNF receptor-associated death domain & TRADD & TNF ligand, TNFA, cachectin \\
Poly (ADP-ribose) polymerase & PARP & ADP ribosyl transferase, ADPRT1, PPOL \\
Spectrin alpha chain & Alpha fodrin & Alpha-II spectrin, fodrin alpha chain \\
Nuclear mitotic apparatus protein & NuMA & SP-H antigen \\
Caspase-activated DNAse & CAD & DNA fragmentation factor subunit beta, DFF-40, caspase- \\
& & activated nuclease, CPAN \\
Inhibitor of CAD & ICAD & DNA fragmentation factor subunit alpha, DFF-45 \\
\hline
\end{tabular}

\section{Perforin/granzyme Pathway (Cytotoxic T} lymphocytes mediated apoptosis):

Cytotoxic T lymphocytes (CTLs) are able to kill target cells via the extrinsic pathway and the FasL/FasR interaction ${ }^{22}$. They are also able to exert their cytotoxic effects on tumor cells and virus-infected cells via a novel pathway called perforin/granzyme pathway. This involves secretion of the transmembrane pore-forming molecule perforin with a subsequent exophytic release of cytoplasmic granules through the pore and into the target cell ${ }^{23}$. The serine proteases granzyme A and granzyme B are the most important component within the granules.

Granzyme B activates procaspase 10 and cleaves factors like Inhibitor of Caspase Activated DNAse (ICAD) ${ }^{24}$. Studies also have shown that granzyme B utilizes mitochondrial pathway for amplification of the death signal by specific cleavage of Bid and induction of cytochrome c release ${ }^{25,26}$. Apart from these granzyme B also directly activate caspase 3 so it bypasses upstream signalling pathway and their by directly induces the execution phase of apoptosis.

Granzyme A is also important in cytotoxic $\mathrm{T}$ cell induced apoptosis and activates caspase independent pathways. Granzyme A protease cleaves the SET complex [Protects chromatin and DNA structure] ${ }^{27}$ and thereby releases inhibition of
NM23-H1 (Nucleosome assembly protein SET, inhibits NM23-H1) leads apoptotic DNA degradation. These suggest that inactivation of this complex by granzyme a most likely also contributes to apoptosis by blocking the maintenance of DNA and chromatin structure integrity.

\section{Inhibitors of apoptosis:}

Caspases are subject to transcriptional regulation and post-translational modifications ${ }^{28}$. In addition, the conserved IAP family of proteins can potently inhibit the enzymatic activity of active caspases and can permanently remove caspases through the ubiquitylation mediated proteasome pathway ${ }^{21,29}$ 30.

The IAP proteins, which were originally identified in the genome of baculovirus on the basis of their ability to suppress apoptosis in infected host cells ${ }^{31}$, have also been found in mammals and fruit flies 21,32 , but not in nematodes. There are eight mammalian IAPs, which include XIAP (X-linked IAP), c-IAP1, c-IAP2, ML-IAP (melanoma IAP)/Livin, ILP2 (IAP-like protein-2), NAIP (neuronal apoptosis-inhibitory protein), Bruce/Apollon and survivin; and two fruit fly IAPs, which include Diap1 (also known as Thread) and Diap2. In mammals, caspases-3, -7 and -9 are subject to inhibition by IAPs. Interestingly, although caspase- 9 binds to several IAPs, it is 
primarily inhibited by XIAP. By contrast, caspase3 and -7 are inhibited by XIAP and, to a lesser extent, by c-IAP1, c-IAP2 and NAIP. In fruit flies, Diap1 directly inhibits the catalytic activity of Drice and targets Dronc to the ubiquitylation mediated degradation pathway ${ }^{33,34}$.

The hallmark of IAPs is the baculoviral IAP repeat (BIR) domain, an $\sim 80$ aminoacid zinc binding domain 35,36 . XIAP, c-IAP1 and c-IAP2 contain three BIR domains each, and the different BIR domains have distinct functions. In XIAP, the third BIR domain (BIR3) potently inhibits the activity of processed caspase-9, whereas the linker region between BIR1 and BIR2 specifically targets caspase-3 and -7. Survivin, which contains a single BIR domain, does not inhibit caspase activity in vitro. Another single BIR containing IAP, MLIAP/Livin, was reported to inhibit both caspase-3 and $-9^{37}$, although it does not seem to contain the sequence elements that are required for this inhibition. In fruit fly Diap1, the second BIR domain (BIR2) is essential for the negative regulation of Dronc, whereas BIR1 is responsible for inhibiting Drice ${ }^{33,34}$.

\section{Anti apoptotic genes:}

A variety of factors have been demonstrated to antagonize apoptotic pathways, both during physiological events (normal development) and in pathological states (viral infections and cancer). Heavily studied anti apoptotic genes include Bcl-2 and Bcl-XL in mammalian cells, Ced-9 in $C$. elegans, p35 in baculovirus, and E1B19K protein in adenovirus.

Bcl-2 was first identified as part of a common translocation in human follicular lymphoma. A novel category of oncogene, Bcl-2 displayed the unusual property of extending cell survival rather than promoting cell proliferation themselves. Three conserved regions termed Bcl-2-homology regions $(\mathrm{BH} 1, \mathrm{BH} 2$ and $\mathrm{BH} 3)$ are required for protein protein interactions of importance in the regulation of apoptosis. Bcl-2 is capable of dimerizing with a number of related factors which, together with factors related by $\mathrm{BH}$ motifs, comprise a family of apoptotic regulators ${ }^{38}$. Although their mechanisms of action are not well understood, some Bcl-2 family members actually promote apoptosis while others (like Bcl-2 itself) antagonize apoptosis. Bcl2 expression is ubiquitous during embryogenesis, but becomes more restricted in adult tissues associated with long-term survival, such as stem cells and proliferating zones. Bcl-2 knockout mice are surprisingly normal at birth, but with severely impaired lymphoid and kidney development as well as age-related melanocyte loss. In contrast, Bcl-2 over expression targeted to the lymphoid system extends B-cell survival with the persistence of immunoglobulin secretion and frequent B-cell lymphoma. Over expression of Bcl-2 is capable of antagonizing apoptosis triggered by c-Myc or p53. However, Bcl-2 rescues apoptosis without affecting the mitogenic function of c-Myc, nor reversing the growth arrest by $\mathrm{p} 53$, indicating that $\mathrm{Bcl}-2$ acts downstream of c-Myc or p53.

$\mathrm{Bcl}-\mathrm{X}$ is a member of the Bcl-2 family. The Bcl-X gene closely resembles $\mathrm{Bcl}-2$, and functions to regulate cell death. Bcl-X transcripts are alternatively spliced into two products: Bcl-XL, the long (L) form and Bcl-LS, the short (S) one. BclXL has an open reading frame of 233 amino acids and has domains similar to those of Bcl-2; Bcl-XS encodes a protein of 170 amino acids with deletion of the Bcl-2 homology domains $\mathrm{BH} 1$ and $\mathrm{BH} 2$. $\mathrm{Bcl}-\mathrm{XL}$ acts to inhibit apoptosis similarly to $\mathrm{Bcl}-2$, whereas Bcl-XS antagonizes the actions of $\mathrm{Bcl}-2$ and Bcl-XL and is proapoptotic. Bcl-XL antagonizes apoptosis in a variety of tissues. BclXL knockout mice die near embryonic day 13, confirming the important role of this antiapoptotic gene in development.

The three-dimensional X-ray crystal structure of $\mathrm{Bcl}-\mathrm{X}$ resembles that of the pore-forming domain of bacterial toxins. Recent study shows that Bcl-X can be embedded into either synthetic lipid vesicles or planar lipid bilayers, and constitutes a $\mathrm{pH}$ sensitive and cation-selective ion-conducting channel at physiological $\mathrm{pH}$.

It is therefore suggested that Bcl-X may sustain cell survival by regulating the permeability of intracellular membranes, or by maintaining mitochondrial membrane potential. Bax is another proapoptotic member of the Bcl-2 family. It and other proapoptotic Bcl-2 family members may act as heterodimer partners of Bcl-2 or Bcl-XL, and 
counteract their ability to protect cell death. Several of the proapoptotic Bcl-2 family members have been connected with other upstream regulators of the apoptosis cascade. Bax expression has been shown to be transcriptionally up regulated by p53 in certain contexts. Another proapoptotic Bcl-2 family member, Bad, is directly phosphorylated by the phosphatidylinositol 3-kinase target Akt, a potentially important regulator of survival signals emanating from the cell membrane. Bid, a third proapoptotic Bcl-2 family member, was recently found to be a direct target of the Fas signaling pathway, linking this extracellular death trigger to regulation of mitochondrial events and caspase activity.

\section{Assays for apoptosis:}

There are various techniques to detect and study of apoptosis. Light and electron microscopy are very useful for this process. Due to lack of cellular synchronization in apoptosis and as apoptotic cells are rapidly engulfed through phagocytosis. Based on methodology apoptosis assays can be classified into different groups.

1. Membrane alterations

2. Changes in Mitochondria

3. Cytomorphological alterations

4. DNA fragmentation

\section{Membrane alterations:}

At the time of apoptosis the lipid phosphatidylserine translocates from the inner to the outer leaflet of the plasma membrane. Annexin $\mathrm{V}$ is a calcium-dependent protein that preferentially binds phosphatidylserine with high affinity. If it is conjugated to a fluorescent tag, Annexin V can be used to detect this early cell surface change of apoptosis ${ }^{39}$. The disadvantage is that the membranes of necrotic cells are labeled as well.

\section{Changes in Mitochondria:}

Dysfunction of mitochondria occurs during apoptosis and it is accompanied by a decreased membrane potential. Mitochondrial assays and cytochrome $\mathrm{c}$ release allow the detection of changes in the early phase of the intrinsic pathway. Membrane potential assays test the mitochondria's ability to concentrate a cationic dye using its proton gradient ${ }^{40}$. The detection of released cytochrome $\mathrm{c}$ is specific for all stages of apoptosis.

\section{Cytomorphological alterations:}

The morphological events of apoptosis are rapid and the fragments are quickly phagocytized, considerable apoptosis may occur in some tissues before it is histologically apparent. This method detects the later events of apoptosis, so cells in the early phase of apoptosis will not be detected.

\section{DNA fragmentation:}

Chromatin condensation and DNA fragmentation is one of the apoptotic hallmark. At the late stage of apoptosis, caspase activated endonucleases break the double-stranded DNA. The DNA laddering techniques used to visualize the endonucleases cleavage products of apoptosis ${ }^{41}$. The TUNEL (Terminal dUTP Nick End-Labeling) method is used to assay the endonucleases cleavage products by enzymatically end labelling the DNA strand breaks ${ }^{42}$.

\section{Caspases:}

The apoptotic process leads to cytoskeletal and nuclear proteolytic digestion as result of activation of a family of aspartate specific protease known as Caspases. Ten enzymes of the human interleukin$1 \beta$ - converting enzyme (ICE/CED-3) super family are collectively known as Caspases. These enzymes are phylogenetically classified into two subfamilies. (1) ICE related enzymes (2) CED-3 related enzymes.

ICE-related enzymes: caspase 1 (ICE), caspase 4 (ICE-II, TX,ICH-2), caspase 5 (ICE-III, TY) and CED-3 related enzymes: caspase 2 (ICH-1, mNEDD-2), caspase 3 (CPP32, APONAIN, YAMA), caspase 6 (MCH2), caspase 7 (MCH3, ICE-LAP3, CMH-1), caspase 8 (MACH, FLICE, MCH5), caspase 9 (ICE-LAP6, MCH6), and caspase $10(\mathrm{MCH} 4)^{43}$.

Each caspase is synthesized as enzyme precursors consisting of N-terminal domains and two subunits. Active heterodimeric proteins are assembled from these subunits. Inactive caspases are activated by proteolytic processing during apoptosis ${ }^{44}$, which may serve as a signal for apoptosis 45 . 
Initiator and Executioner caspases: Architecture and Activation:

Initiator caspases: are single chains, consist of death domain at the $\mathrm{N}$-terminus and followed by a catalytic domain, which is divided into a large and small subunit and a long loop region between the subunits. But initiator caspases, such as caspase-9, show full activity in their uncleaved forms. Caspase 9 activity is regulated by dimerization, instead of by cleavage. Activated caspase 9 bind to an oligomeric platform (apoptosome) via adaptor domains (such as CARD, caspase recruitment domain) and results in an induced proximity of the catalytic domains of initiator caspases (Fig. 3).

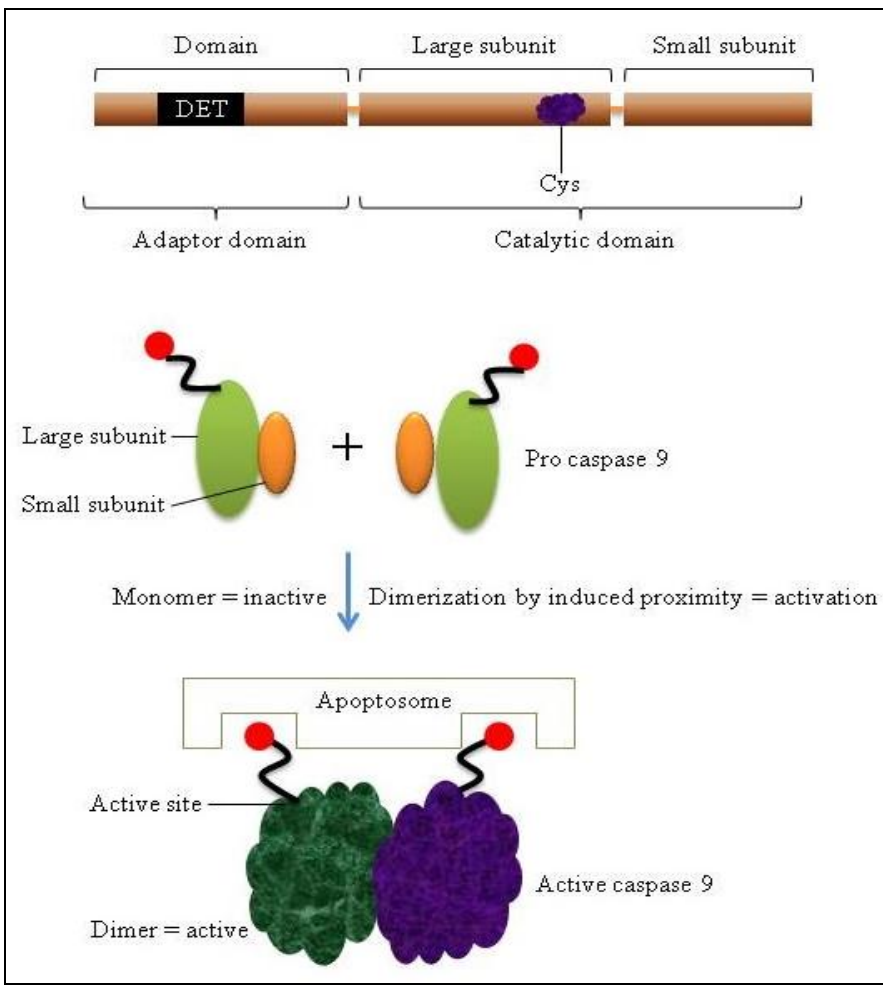

FIGURE 3: INITIATOR CASPASES: ARCHITECTURE AND ACTIVATION

Executioner caspases are initially expressed as single-chain proteins and activated by cleavage. An executioner caspase is typically cleaved twice, leading to the release of a short $\mathrm{N}$-terminal peptide. The activation cleavage divides the catalytic unit into a large and small subunit. The executioner caspases are constitutive dimers of two catalytic units and their active-site cysteine residues are indicated in red. An executioner caspase (caspase7) preactivation and post activation cleavage. Cleavage releases strains on surface loops (red and orange) and the chains rearrange. The newly formed termini of large and small subunit (orange) interact with each other across the other catalytic unit, and with the red loops to nicely align the substrate-binding pockets at the bottom of the active-site cleft. This results in a highly active caspases (Fig 4).

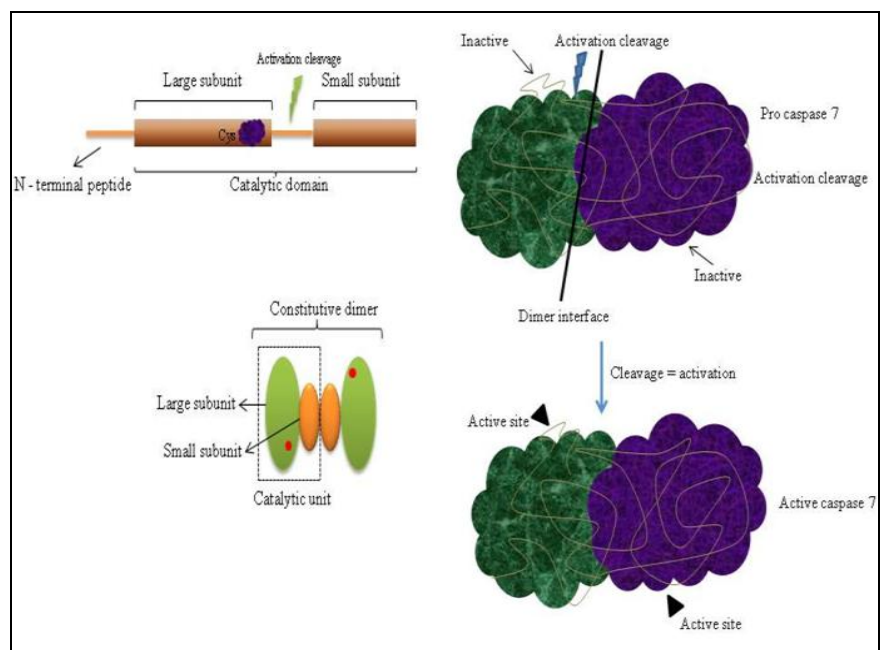

FIGURE 4: EXECUTIONER CASPASES: ARCHITECTURE AND ACTIVATION.

\section{Clinical implications:}

The mechanisms involved in disordered apoptosis were unravelled, suggesting that alterations in control of cell death or survival are implicated in pathogenesis of a variety of human diseases including cancer and many other chronic diseases ${ }^{3}$. Deficient apoptosis is associated with cancer, autoimmune disorders and viral infections, while excessive apoptosis is associated with ischemic injury (myocardial infarction, stroke, AIDS, neurodegenerative disease, sepsis and multiple organ dysfunction syndromes) $4,46,47$.

\section{Apoptosis and cardiovascular system:}

Apoptosis has been documented in myocardial ischemia, reperfusion and infarction, heart failure induced by rapid ventricular pacing or coronary microemboli, several models of pressure overload hypertrophy and ageing. Arrhythmias may be associated with deficient apoptosis during cardiac development.

Normally after birth there is apoptosis of the small round pacemaker cells in the central and lower part of the atrioventricular node (AV) node. Delay or failure of apoptosis in these cells may lead to lifethreatening arrhythmias that may resolve 
spontaneously. Excessive apoptosis can lead to sudden death ${ }^{48}$. Patients with long QT syndrome have increased rates of apoptosis in the sinus node 49 .

Apoptosis has also been demonstrated in hypertrophic cardiomyopathy (HCM) and dilated cardiomyopathy (DCM) as evidenced by activated apoptotic pathway components and caspase activity 50 . Many of the signaling pathways activated with increased cardiomyocyte stretch are implicated in regulation of apoptosis, including Akt and adrenergic stimulation. Hyperactivation of $\beta$ adrenergic signaling pathways and cytochrome c release from mitochondria are associated with increased apoptosis and fibrosis ${ }^{48}$. Additionally, evidence for increased signaling through Fas receptors by up-regulation of TNF is common in heart failure, and the degradation of inhibitors of Fas receptor signaling such as cFlip is another mechanism by which apoptosis may occur ${ }^{51}$.

Studies from human heart biopsies reveal that greater cell loss is observed in the early stages of cardiomyopathy, suggesting that anti apoptotic pathways are up regulated after cell loss has been initiated ${ }^{46}$. It follows that tight regulation of apoptosis is required for proper cardiac adaptation and that disruption of these pathways can have irreversible consequences in the heart.

\section{Apoptosis and reproductive system:}

Apoptosis is continually inhibited in many tissues of the reproductive system owing to the presence of trophic hormones from the pituitary, gonads and uterus. When the hormones are removed, the tissues undergo atrophy. Ovarian follicles undergo growth or atresia in response to cyclic changes in luteinizing hormone and follicle stimulating hormone; the endometrium, breast and prostate are dependent on the steroid hormones and regress when these are removed ${ }^{52}$.

\section{Apoptosis and male fertility:}

Numerous studies have shown a relationship between apoptosis markers and male infertility ${ }^{53}$. Phosphatidylserine externalization and DNA fragmentation were shown to occur more frequently in spermatozoa that were positive for active caspase-3. Apoptosis markers appeared significantly higher in low-motility spermatozoa as compared with high-motility spermatozoa 54 . Similarly, percentage of apoptosis has been shown to be negatively correlated with motility and normal morphology and positively associated with sperm tail defects ${ }^{55}$. These findings suggest a possible relationship between increased caspase activity, DNA damage, phosphatidylserine externalization and sperm quality in terms of routine parameters.

\section{Apoptosis and gastrointestinal tract:}

Apoptosis is essential for maintaining the normal gut epithelial function. Gastrointestinal diseases may be associated with excessive or defective apoptosis. Dysregulated apoptosis is seen in a number of pathological conditions of the gastrointestinal tract, like autoimmune liver disease, inflammatory bowel disease, ischaemiareperfusion, liver cancer, chronic viral hepatitis, alcohol liver disease and primary biliary cirrhosis.

Progressive inhibition of apoptosis appears to be involved in the pathogenesis of gastrointestinal neoplasia, in particular colorectal cancer ${ }^{56}$. Nonsteroidal anti-inflammatory drugs, which inhibit COX-1 and COX-2, can prevent up-regulation of Bcl-2 by prostaglandins and prevent colorectal carcinoma. The protective effects of dietary fibre may be via its fermentation by bacteria in the colon to form short-chain fatty acids which promote $\operatorname{apoptosis}^{57}$.

CONCLUSIONS: Apoptosis is an exceedingly complicated phenomenon, characterized by specific morphological and biochemical features in which caspase activation plays a central role. Apoptosis plays a significant role in survival by maintaining the homeostasis in multicellular organisms as well as in the management of many diseases, since malfunctioning of apoptotic pathway may cause several human diseases like cancer, neurodegenerative as well as several types of autoimmune disorder. Understanding the mechanisms of apoptosis and other variants of programmed cell death, at the molecular level provides deeper insight into various disease processes and may thus influence therapeutic strategy. 


\section{REFERENCES:}

1. Osborn BA and Schwartz LM: Essential genes that regulate apoptosis. Trends in Cell Biology 1994; 4: 394398.

2. Steller H: Apoptosis. Science 1995; 267: 1443-1449.

3. Thompson CB: Apoptosis in the pathogenesis and treatment of disease. Science 1995; 267: 1456-1462.

4. Saikumar P, Dong Z, Mikhailov V, Denton M, Weinberg JM and Venkatachalam MA: Apoptosis: definition, mechanisms, and relevance to disease. American Journal of Medicine 1999; 107: 489-506.

5. Norbury CJ and Hickson ID: Cellular responses to DNA damage. Annual review of pharmacology and toxicology 2001; 41: 367-401.

6. Solary E, Bertrand R, and Pommier Y: Differential induction of apoptosis in undifferentiated and differentiated HL-60 cells by DNA topoisomerase I and II inhibitors. Blood 1993; 81: 1359-1368.

7. Hacker G: The morphology of apoptosis. Cell and Tissue Research 2000; 301: 5-17.

8. Kerr JF, Wyllie AH and Currie AR: Apoptosis: a basic biological phenomenon with wide-ranging implications in tissue kinetics. British Journal of Cancer 1972; 26: 239-57.

9. Peitch M, Polzaz B and Stephan H: Characterization of the endogenous deoxyribonuclease involved in nuclear DNA degradation during apoptosis (Programmed Cell Death). EMBO Journal 1993; 12: 371-377.

10. O'Connor L, Huang DC and O'Reilly LA: Apoptosis and cell division. Current Opinion in Cell Biology 2000; 12: 257-263.

11. Wolf BB and Green DR: Apoptosis: letting slip the dogs of dispatch war. Current Biology 2002; 12: 177-179.

12. Igney FH and Krammer PH: Death and anti-death: tumour resistance to apoptosis. Nature Reviews Cancer 2002; 2: 277-88.

13. Martinvalet $\mathrm{D}$, Zhu $\mathrm{P}$ and Lieberman J: Granzyme A induces caspase independent mitochondrial damage, a required first step for apoptosis. Immunity 2005; 22: 35570.

14. Ashkenazi A and Dixit VM: Death receptors: signaling and modulation. Science. 1998; 281: 1305-1308.

15. Yang X, Khosravi FR and Chang HY: A novel Fasbinding protein that activates JNK and apoptosis. Cell 1997; 89: 1067-1076.

16. Peter EM and Krammer PH: The CD95 (Apo-1/Fas) DISC and beyond. Cell Death \& Differentiation 2003; 10: 26-35.

17. Foo RS, Mani K and Kitsis RN: Death begets failure in the heart. Journal of Clinical Investigation 2005; 115: 565571.

18. Hussein MR, Haemel AK and Wood GS: Apoptosis and melanoma: molecular mechanisms. Journal of Pathology 2003; 199: 275-288.

19. Acehan D, Jiang X, Morgan DG, Heuser JE, Wang X and Akey CW: Three dimensional structure of the apoptosome - implications for assembly, procaspase-9 binding and activation. Molecular Cell 2002. 9: 423-432.

20. Verhagen AM, Ekert PG, Pakusch M, Silke J, Connolly LM, Reid GE, Moritz RL, Simpson RJ and Vaux DL: Identification of DIABLO a mammalian protein that promotes apoptosis by binding to and antagonizing IAP proteins. Cell 2000; 102: 43-53.

21. Salvesen GS and Duckett CS: IAP proteins: blocking the road to death's door. Nature Reviews Molecular Cell Biology 2002; 3: 401-410.

22. Brunner T, Wasem C, Torgler R, Cima I, Jakob S and Corazza N: Fas (CD95/Apo-1) ligand regulation in $\mathrm{T}$ cell homeostasis, cell-mediated cytotoxicity and immune pathology. Seminars in Immunology 2003; 15: 167-76.

23. Trapani JA and Smyth MJ: Functional significance of the perforin/granzyme cell death pathway. Nature Reviews Immunology 2002; 2: 735-47.

24. Sakahira H, Enari $M$ and Nagata S: Cleavage of CAD inhibitor in CAD activation and DNA degradation during apoptosis. Nature. 1998; 391: 96-9.

25. Barry M and Bleackley RC: Cytotoxic T lymphocytes: all roads lead to death. Nature Reviews Immunology 2002; 2: 401-9.

26. Russell JH and Ley TJ: Lymphocyte-mediated cytotoxicity. Annual Review of Immunology 2002; 20: 323-70.

27. Lieberman $\mathbf{J}$ and Fan $\mathrm{Z}$ : Nuclear war: the granzyme Abomb. Current Opinion in Immunology 2003; 15: 553-9.

28. Earnshaw WC, Martins LM and Kaufmann SH: Mammalian caspases: structure, activation, substrates, and functions during apoptosis. Annual Review of Biochemistry 1999; 68: 383-424.

29. Alnemri ES, Livingston DJ, Nicholson DW, Salvesen G, Thornberry NA, Wong WW and Yuan J: Human ICE/CED-3 protease nomenclature. Cell 1996; 87: 171.

30. Deveraux QL and Reed JC: IAP family proteinssuppressors of apoptosis. Genes \& Development 1999; 13: 239-252.

31. Miller LK: An exegesis of IAPs: salvation and surprises from BIR motifs. Trends in Cell Biology 1999; 9: 323328.

32. Hay BA: Understanding IAP function and regulation: a view from drosophilia. Cell Death \& Differentiation 2000; 7: 1045-1056.

33. Ni CZ, Li C, Wu JC, Spada AP and Ely KR: Conformational restrictions in the active site of unliganded human caspase-3. Journal of Molecular Recognition 2003; 16: 121-124.

34. Chai J, Yan N, Huh JR, Wu JW, Li W, Hay BA and Shi Y: Molecular mechanism of reaper-grim-hid mediated suppression of DIAP1-dependent dronc ubiquitination. Nature Structural \& Molecular Biology 2003; 10: 892-898.

35. Sun C, Cai M, Gunasekera AH, Meadows RP, Wang H, Chen J, Zhang H, Wu W, Xu N, Ng SC and Fesik SW: NMR structure and mutagenesis of the inhibitor of apoptosis protein XIAP. Nature. 1999; 401: 818-822.

36. Hinds MG, Norton RS, Vaux DL and Day CL: Solution structure of a baculoviral inhibitor of apoptosis (IAP) repeats. Nature Structural Biology 1999; 6: 648-651.

37. Kasof GM and Gomes BC: Livin a novel inhibitor of apoptosis protein family member. Journal of Biological Chemistry 2001; 276: 3238-3246.

38. Chao DT and Korsmeyer SJ: BCL-2 family: regulators of cell death. Annual Review of Immunology 1998; 16: 395419.

39. Apoptosis detection guide. Enzo Life Sciences Inc. International edition. 2010: 1-14.

40. Poot $M$ and Pierce RH: Detection of changes in mitochondrial function during apoptosis by simultaneous staining with multiple fluorescent dyes and correlated multiparameter flow cytometry. Cytometry. 1999; 35: 311 7.

41. Wyllie AH: Glucocorticoid-induced thymocyte apoptosis is associated with endogenous endonuclease activation. Nature. 1980; 284: 555-6.

42. Kressel M and Groscurth P: Distinction of apoptotic and necrotic cell death by in situ labelling of fragmented DNA. Cell and Tissue Research 1994; 278: 549-56. 
43. Rodriquez I, Matsuura K and Khatib A: A bcl-2 transgene expressed in hepatocytes protects mice from fulminant liver destruction but not from rapid death induced by antifas antibody injection. Journal of Experimental Medicine 1996; 183: 1031-1037.

44. Nicholson DW, Green DR and Kluck RM: The release of cytochrome $\mathrm{C}$ from mitochondria: A primary sticite for bcl-2 regulation of apoptosis. Science. 1997; 275: 11321136.

45. Salvesen GS and Dixit VM: Caspases: intracellular signaling by proteolysis. Cell. 1997; 91: 443-446.

46. Zornig M, Hueber AO, Baum W, Evan G: Apoptosis regulators and their role in tumorigenesis. Biochimica et Biophysica Acta 2001; 1551: F1-F37.

47. Hellerstein MK and McCune JM: T cell turnover in HIV-1 disease. Immunity. 1997; 7: 583-9.

48. Narula J, Kharbanda S, Khaw BA: Apoptosis and the heart. Chest. 1997; 112: 1358-62.

49. James TN: Normal and abnormal consequences of apoptosis in the human heart: from postnatal morphogenesis to paroxysmal arrhythmias. Transactions of the American Clinical and Climatological Association 1993; 105: 145-77.

50. Akyurek O, Akyurek N, Sayin T, Dinçer I, Berkalp B, Akyol G, Ozenci M, Oral D: Association between the severity of heart failure and the susceptibility of myocytes to apoptosis in patients with idiopathic dilated cardiomyopathy. International Journal of Cardiology 2001. 80: 29-36.

51. Krown KA, Page MT, Nguyen CD, Zechner V, Gutierrez KL, Comstock CC Glembotski PJ, Quintana RA and
Sabbadini: Tumor necrosis factor alpha-induced apoptosis in cardiac myocytes. Involvement of the sphingolipid signaling cascade in cardiac cell death. Journal of Clinical Investigation 1996; 98: 2854-2865.

52. Gosden R and Spears N: Programmed cell death in the reproductive system. British Medical Bulletin. 1997; 53: 644-61.

53. Zedan H, El-Mekhlafi AW, El-Noweihi AM, Abd El-Azim NE, Mostafa T: Soluble Fas and gonadal hormones in infertile men with varicocele. Fertility and Sterility 2009; 91: 420-424

54. Weng SL, Taylor SL, Morshedi M, Schuffner A, Duran EH, Beebe S, Oehninger S: Caspase activity and apoptotic markers in ejaculated human sperm. Molecular Human Reproduction 2002; 8: 984-991.

55. Chen Z, Hauser R, Trbovich AM, Shifren JL, Dorer DJ, Godfrey-Bailey L and Singh NP: The relationship between human semen characteristics and sperm apoptosis: a pilot study. Journal of Andrology 2006; 27: 112-120.

56. Bedi A, Pasricha PJ, Akhtar AJ, Barber JP, Bedi GC, Giardiello FM, Zehnbauer BA, Hamilton SR, Jones RJ: Inhibition of apoptosis during development of colorectal cancer. Cancer Research 1995; 55: 1811-16.

57. Pritchard DM and Watson AJM: Apoptosis and gastrointestinal pharmacology. Pharmacological Therapeutics. 1996; 72: 149-69.

58. Human Protein Reference Database http://www.hprd.org/.

59. ExPASy Proteomics Serverhttp://ca.expasy.org/.

How to cite this article:

Kar B and Sivamani S: Apoptosis: Basic Concepts, Mechanisms and Clinical Implications. Int J Pharm Sci Res 2015; 6(3): 940-50.doi: 10.13040/IJPSR.0975-8232.6 (3).940-50.

All @ 2013 are reserved by International Journal of Pharmaceutical Sciences and Research. This Journal licensed under a Creative Commons Attribution-NonCommercial-ShareAlike 3.0 Unported License.

This article can be downloaded to ANDROID OS based mobile. Scan QR Code using Code/Bar Scanner from your mobile. (Scanners are available on Google Playstore) 